\title{
Room-Temperature Mid-Wave Infrared Guided-Mode Resonance Detectors
}

This paper was downloaded from TechRxiv (https://www.techrxiv.org).

LICENSE

CC BY 4.0

SUBMISSION DATE / POSTED DATE

27-02-2022 / 03-03-2022

\section{CITATION}

Kamboj, Abhilasha; Nordin, Leland; Woolf, David; Wasserman, Daniel (2022): Room-Temperature Mid-Wave Infrared Guided-Mode Resonance Detectors. TechRxiv. Preprint.

https://doi.org/10.36227/techrxiv.19245876.v1

$\mathrm{DOI}$

10.36227/techrxiv.19245876.v1 


\title{
Room-Temperature Mid-Wave Infrared Guided-Mode Resonance Detectors
}

\author{
Abhilasha Kamboj, Leland Nordin, David Woolf, and Daniel Wasserman
}

\begin{abstract}
We demonstrate room temperature mid-wave infrared detectors with high peak detectivity. Our detector structures consist of type-II superlattice $\mathrm{nBn}$ detectors with ultra-thin $(\sim 250 \mathrm{~nm})$ absorbers, integrated into an all-epitaxial guidedmode resonance architecture. The resulting devices show strong spectral selectivity, controllable by choice of grating period, and low dark currents. We achieve room temperature peak specific detectivity of $D * \approx 1.2 \times 10^{10} \mathrm{~cm} \sqrt{\mathrm{Hz}} / \mathrm{W}$ at a wavelength of $\lambda=4.4 \mu \mathrm{m}$ and $D^{*} \approx 1 \times 10^{10} \mathrm{~cm} \sqrt{\mathrm{Hz}} / \mathrm{W}$ at a wavelength of $\lambda=4.7 \mu \mathrm{m}$, for grating periods of $\Lambda=1.6 \mu \mathrm{m}$ and $\Lambda=1.8 \mu \mathrm{m}$, respectively. The presented all-epitaxial devices offer a unique approach to efficient room temperature mid-wave infrared detection with strong spectral and polarization selectivity.
\end{abstract}

Index Terms-Photodetector, mid-infrared, superlattice, sensor.

\section{INTRODUCTION}

$\mathbf{T}$ HE mid-wave infrared (MWIR, $3-5 \mu \mathrm{m}$ ) is a technologically vital wavelength range with potential applications for gas sensing, free space communication, and thermal imaging of hot objects. All of the above benefit from, or require, sensitive, high-operating-temperature (HOT) photodetectors. The current state of the art MWIR detectors are generally $\mathrm{InSb}$ or $\mathrm{HgCdTe}$ (MCT) photodiode detectors. $\mathrm{InSb}$ detectors often require cryogenic cooling in order to maintain acceptable dark currents, while MCT detectors suffer from growth non-uniformity and concerns regarding toxicity of their constituent materials. Recently, there has been significant interest in III-V type-II superlattice (T2SL) materials for midIR detection applications. T2SLs comprise alternating layers of semiconductor materials having either staggered or brokengap band offsets. For thin layers, the overlap of electron (hole) wavefunctions leads to the formation of minibands in the conduction (valence) band, where the energy spacing between the ground-state electron and hole minibands can be smaller than the band gap of either constituent material. T2SLs thus provide a unique infrared material system, with an effective band-gap controlled by a choice of layer materials and thicknesses. T2SLs have been predicted to benefit from reduced Auger recombination [1] and therefore lower dark current, and can be integrated into a range of photodetector architectures, including p-i-n-junction photodiodes [2], bariode devices (such as $n B n$ or CBIRD) [3], [4] or even phototransistors [5]. However, the spatial offset of electron and hole wavefunctions

A. Kamboj, L. Nordin, and D. Wasserman are with the Department of Electrical and Computer Engineering, University of Texas at Austin, Austin, TX, 78758 USA e-mail:dw@utexas.edu.

D. Woolf is with Physical Sciences Inc.

Manuscript received ; revised in T2SL materials results in a reduced absorption coefficient, when compared to bulk materials [6]. Countering this reduced absorption requires thicker detectors, which, consequently, will increase detector dark current (noise) and reduce detector response times [7].

Accordingly, there has been interest in incorporating T2SL detectors into resonant optical structures in order to enhance the detector material's effective absorption coefficient [8][10]. Recently, it was demonstrated that MWIR T2SL nBn detectors could be integrated into guided-mode resonance (GMR) structures to allow for spectrally-selective detection with external quantum efficiency (EQE) of over 50\% [11]. The GMR structure is effectively a dielectric waveguide comprising a planar layer of high-index material patterned with a sub-wavelength grating, sandwiched between two low-index layers such that there is significant contrast in refractiveindex between layers [12]. Incident light can be coupled into the waveguide at certain distinct frequencies (for a given polarization and incidence angle), after which it is scattered back out into free space. The constructive/destructive interference between incident, transmitted, and scattered light results in sharp dips/peaks in transmission/reflection, making these structures of significant interest for light filtering applications across a wide range of wavelengths [13]-[15]. The initial $\left(T_{o p}=200 \mathrm{~K}\right)$ demonstration of MWIR GMR T2SL detectors leveraged a low-index highly doped semiconductor layer beneath an $\mathrm{nBn}$ detector to form the waveguide structure, with the grating etched into a sacrificial GaSb layer grown above the $\mathrm{nBn}$ detector. These detectors, while showing excellent performance up to $\mathrm{T}=200 \mathrm{~K}$, degraded with increasing temperature and demonstrated anomalously large turn-on voltages (problematic for detector array operation). In the current work, we demonstrate room temperature $(\mathrm{RT}=296 \mathrm{~K})$ operation of redesigned MWIR GMR detectors, with enhanced EQE (EQE $\approx 60 \%$ ), and specific detectivity, $D^{*}>1 \times 10^{10} \mathrm{~cm} \sqrt{\mathrm{Hz}} / \mathrm{W}$. In addition, our detectors show turn-on voltages less than $-0.2 \mathrm{~V}$. The presented high EQE and high-detectivity devices offer a potentially transformative detector architecture for a range of vital mid-IR applications.

\section{Device Design And Growth, Experimental SET-UP}

The improved performance of the MWIR GMR photodetectors, compared to previous devices [11], result from changes to the device design and T2SL materials employed. First, the decrease in turn-on voltage was achieved by thinning our barrier layer, and p-doping the contact layer to create a tunneljunction to the GaSb. The increase in $T_{o p}$ results from the 


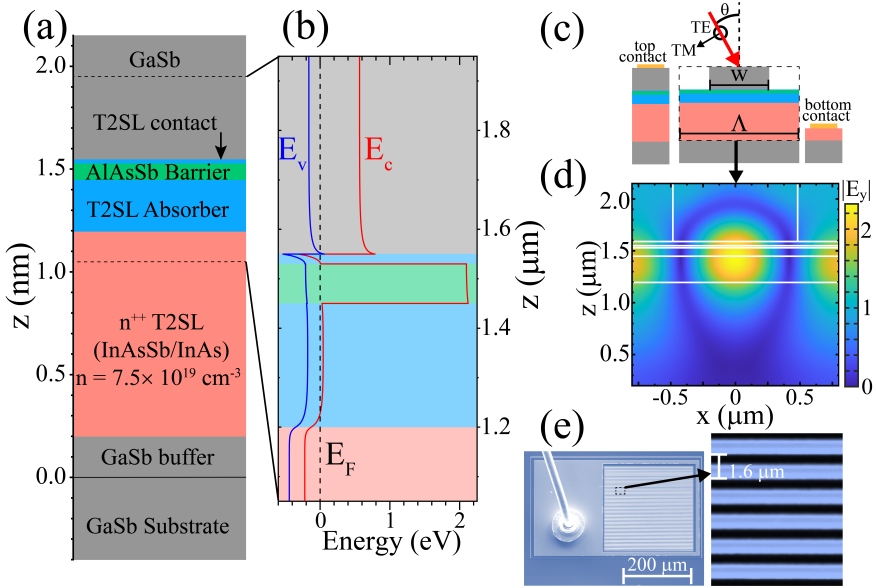

Fig. 1. (a) Growth stack for MWIR GMR detector structure and (b) band structure of $\mathrm{nBn}$ detector. (c) Cross sectional schematic of a single period of the GMR detector, with location of top and bottom detector contacts shown on the left and right side, respectively. (d) Electric field profile of MWIR GMR structure for TE polarized incident light of wavelength $\lambda=4.44 \mu \mathrm{m}$. (e) Scanning electron micrograph of fabricated detector structure with expanded view of the $\Lambda=1.6 \mu \mathrm{m}$ gratings.

use of a shorter cut-off wavelength absorber $\left(\lambda_{c o} \sim 5 \mu \mathrm{m}\right)$, which dramatically reduces detector dark current $J_{\text {dark }}$. Our devices were grown on a 2-inch lightly n-doped GaSb substrate in a Varian Gen II molecular beam epitaxy (MBE) system, beginning with a $200 \mathrm{~nm}$ n-doped $\mathrm{GaSb}$ buffer, followed by the device growth stack. First, a $1 \mu \mathrm{m}$ thick highly doped $n^{++}$ InAs/InAs ${ }_{0.49} \mathrm{Sb}_{0.51} \mathrm{~T} 2 \mathrm{SL}$ layer acts as the bottom cladding of the waveguide. Above the highly-doped T2SL is grown the $\mathrm{nBn}$ detector structure, consisting of a $250 \mathrm{~nm}$ thick InAs/InAs ${ }_{0.49} \mathrm{Sb}_{0.51}$ T2SL (12 ML Period) absorber, an 80 $\mathrm{nm}$ thick unintentionally doped (UID) $\mathrm{AlAs}_{0.08} \mathrm{Sb}_{0.92}$ barrier and a $20 \mathrm{~nm}$ contact layer of the same T2SL material as the absorber. The structure is capped with a $600 \mathrm{~nm}$ thick UID GaSb layer. All superlattice layers have the same composition but different doping concentrations. The absorber is unintentionally doped (lightly n-doped), whereas the contact is p-doped to a concentration of $10^{18} \mathrm{~cm}^{-3}$. The $\mathrm{n}^{++}$T2SL layer serves as a low-index bottom cladding layer, where the highdoping of the material reduces the permittivity according to the Drude formalism, which provides an accurate mechanism for calculating the dielectric response of highly-doped semiconductors in the mid-IR [16]. Although it uses the same T2SL as the detector absorber, state-filling and ultra-fast non-radiative recombination times [17] in the $\mathrm{n}^{++}$layer ensures that it does not contribute to the optical response of the detector. The top GaSb layer serves as the high-index grating material. The device layer stack and band structure are shown in Fig. 1(a-b).

Figures 1(c) \& (e) show the cross-sectional schematic and scanning electron micrograph (SEM) of a fabricated device, respectively. Detector fabrication begins with large area mesas deep-etched to the $\mathrm{n}^{++}$layer. Contacts $(\mathrm{Ti}(40 \mathrm{~nm}) / \mathrm{Pt}(15 \mathrm{~nm}) / \mathrm{Au}(180 \mathrm{~nm}))$ are patterned and deposited, with a top window contact on the etched mesa, and the bottom contact on the exposed $\mathrm{n}^{++}$layer. Gratings of period $\Lambda=1.6$ and $1.8 \mu \mathrm{m}$ are etched into the $\mathrm{GaSb}$, to within 10's of $\mathrm{nm}$ of the $\mathrm{nBn}$ contact layer, with reactiveion etching (RIE) using a patterned $\mathrm{SiN}_{x}$ hard mask. Control samples are fabricated without gratings, but with the $\mathrm{GaSb}$ top layer etched to the same depth as the gratings. The fabricated samples are mounted and wire-bonded to leadless chip carriers (LCC). Temperature-dependent dark current-voltage measurements are made with a copper shield (to block any external light from the device), attached to the cold-finger upon which the LCC is mounted. The GMR detector spectral response is measured using a Bruker Vertex 80v Fouriertransform infrared (FTIR) spectrometer, and normalized to the flat spectral response of a pyroelectric detector, to account for the spectral weighting of the FTIR output beam. The detector signal resulting from the emission from a calibrated $1000^{\circ} \mathrm{C}$ blackbody source, modulated by an optical chopper, and passing through a variable aperture and a $3.6 \mu \mathrm{m}$ bandpass filter, is then measured. We then calculate the wavelength dependent EQE by scaling the normalized FTIR spectra by the responsivity measured at $\lambda=3.6 \mu \mathrm{m}$.

Detector response is modeled using rigorous coupled-wave analysis (RCWA) [18], which solves for reflection and transmission, as well as the optical fields in our structures, as a function of wavelength, incidence angle and polarization. The $\mathrm{n}^{++}$T2SL is modelled as a Drude metal with plasma wavelength $\lambda_{p}=5.7 \mu \mathrm{m}$ and scattering rate $\gamma=1 \times 10^{13} \mathrm{rad} \mathrm{s}^{-1}$. The real part of the permittivity for the T2SL absorber, barrier, and contact layer are determined from a weighted average of their constituent materials. The complex permittivity of our T2SL is estimated by fitting the experimental spectral response of our control device to our RCWA results, using the detector absorption coefficient $\alpha(\lambda)$ as our fitting parameter, assuming an anisotropic absorption coefficient where only the in-plane components of $\alpha$ are non-zero [19]. We use the simulated field profiles to model the detector EQE by integrating the Poynting vector, $S$, over each interface and taking the difference between adjacent interfaces. Because the typical diffusion length associated with T2SL materials is significantly longer than our absorber thickness [20], we assume a $100 \%$ collection efficiency of photo-generated charge carriers. The model considers light directly transmitted through the detector and reflected from the backside of the wafer. The lightly ndoped GaSb substrate absorption coefficient is modeled with a combination of electron free carrier absorption and inter-valley conduction band absorption [21]. Our simulations suggest that $\sim 2 \%$ of our peak EQE results from backside reflection.

\section{RESUlTS AND DISCUSSION}

Figure 2(a) shows the dark current as a function of temperature from a representative $\Lambda=1.8 \mu \mathrm{m}$ grating detector. A clear turn-on is seen in the devices at a reverse bias as low as $-0.1 \mathrm{~V}$. The dark current remains largely constant for reverse biases larger than $-0.2 \mathrm{~V}$, indicative of diffusion current dominating the total dark current. The dark current at $V=-0.2 \mathrm{~V}$, as a function of temperature, is shown in Fig. 2(b), as well as the predicted current for an ideal MCT detector calculated using the so-called "Rule 07" [22], a heuristic often used as a benchmark for mid-IR detector dark 


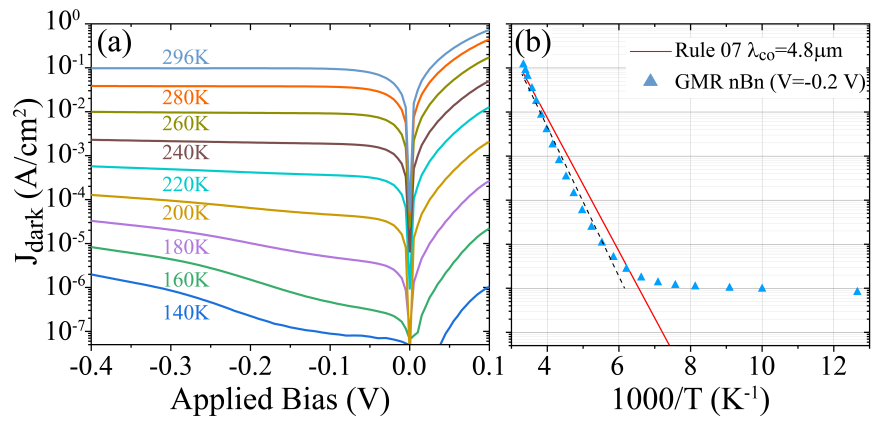

Fig. 2. (a) MWIR GMR $n B n$ detector dark current density, $J_{\text {dark }}$ for temperatures from $140 \mathrm{~K}$ to room temperature $(\mathrm{T}=296 \mathrm{~K})$. At room temperature, $\mathrm{J}_{\text {dark }}$ indicates the detector turn-on at $\sim-0.1 \mathrm{~V}$ and largely voltageindependent dark current above turn-on, as expected for an $\mathrm{nBn}$ detector. (b) Arrhenius plot of our detector dark current (blue triangles) at an applied bias of $V=-0.2 \mathrm{~V}$ as a function of temperature. From this plot, we can extract the effective cut-off wavelength of our detector at room temperature (dashed line, $\lambda_{c o}=4.8 \mu \mathrm{m}$ ). Also plotted is the predicted dark current for an MCT detector with $\lambda_{c o}=4.8 \mu \mathrm{m}$ using the Rule 07 heuristic (red solid line).

currents. While our detector dark currents do not approach the recently demonstrated dark currents from MCT detectors leveraging carrier depletion Auger suppression [23], they are below or comparable to Rule 07 for temperatures above $160 \mathrm{~K}$, and well below those of typical III-V semiconductor MWIR photodetectors [24], [25].

The experimental EQE at $T_{o p}=296 \mathrm{~K}$ for the $\Lambda=1.6 \mu \mathrm{m}$ and $\Lambda=1.8 \mu \mathrm{m}$ grating period detectors, as well as the control sample, are shown in Fig. 3, for TE-polarized incident light. Strong peaks in EQE are seen in both grating period samples. For the $\Lambda=1.6 \mu \mathrm{m}$ sample, peak $\mathrm{EQE}$ is observed at $\lambda \approx 4.4 \mu \mathrm{m}$, reaching a peak value of $\sim 60 \%$, a factor of $\times 7.5$ enhancement over the unpatterned control sample. For the $\Lambda=1.8 \mu \mathrm{m}$ sample, peak EQE is observed at $\lambda=4.7 \mu \mathrm{m}$, reaching a maximum value of over $46 \%$, a factor of $\times 14$ enhancement over the unpatterned control sample. We demonstrate control over peak absorption in our detectors by tuning the detector grating period, allowing for peak response to be designed across the MWIR. Moreover, we observe a substantial enhancement of detector response, with particularly strong enhancement at or near the bandedge of the T2SL absorber. Typically, absorption at the bandedge of a T2SL is weak, and if efficient detection is required at a specific wavelength, $\lambda_{0}$, it is often necessary to use an absorber cutoff wavelength $\left(\lambda_{c o}\right)$ much longer than $\lambda_{0}$. Such an approach comes with trade-offs however, as dark current is exponential in absorber cut-off wavelength, so that specific detectivity $\left(D^{*}\right)$ gains due to increased EQE are offset by increased $J_{\text {dark }}$. The GMR MWIR detector architecture thus allows for a strong enhancement of EQE at or near the absorber bandedge, without having to resort to longer $\lambda_{c o}$, effectively decoupling the dark current/EQE trade-off intrinsic in the majority of MWIR detector materials and architectures.

In figure 4, we present the estimated (TE-polarized) specific detectivity of our room temperature MWIR GMR detectors. Specific detectivity is calculated with the expression, $D^{*}=$ $R_{i} \sqrt{\frac{A}{2 q|I|+4 k_{b} T / R}}$, from the experimentally measured detector current, dynamic resistance, and responsivity $(I, R=d V / d I$,
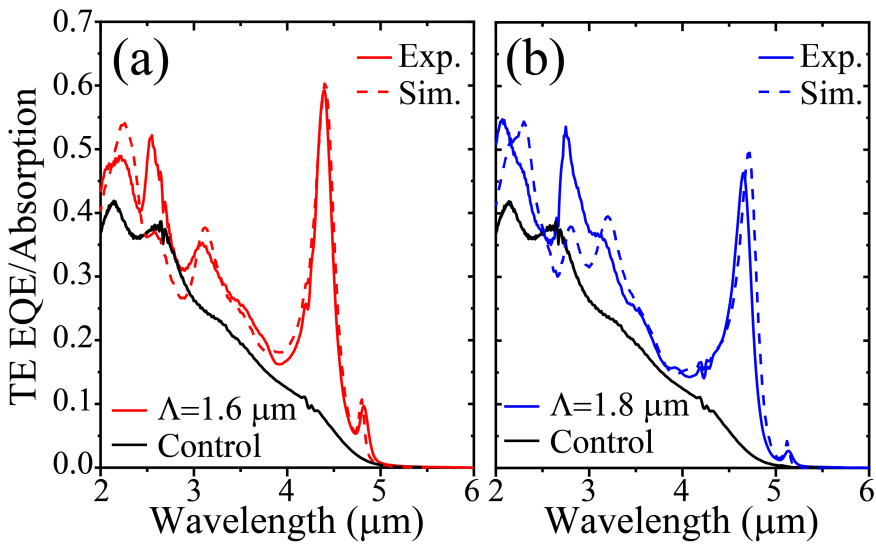

Fig. 3. TE-polarized experimental (solid) and modeled (dashed) external quantum efficiency for the (a) $\Lambda=1.6 \mu \mathrm{m}$ (red) and (b) $\Lambda=1.8 \mu \mathrm{m}$ grating detector devices. The EQE from an unpatterned control sample is shown in (a) and (b) for comparison. Because the absorption coefficient is determined by fitting the control response, the modelled and experimental control EQE overlap.



Fig. 4. Room temperature TE-polarized estimated special detectivity $\left(D^{*}\right)$ of the control (black), and the $\Lambda=1.6 \mu \mathrm{m}$ (red) and $\Lambda=1.8 \mu \mathrm{m}$ (blue) grating detector devices.

and $R_{i}$, respectively), mesa area $A$, electronic charge $q$, Boltzmann's constant $k_{b}$, and temperature $T$. We obtain peak specific detectivity of $1.2 \times 10^{10}$ and $1 \times 10^{10} \mathrm{~cm} \sqrt{\mathrm{Hz}} / \mathrm{W}$ for the $\Lambda=1.6 \mu \mathrm{m}$ and $\Lambda=1.8 \mu \mathrm{m}$ grating-patterned detectors respectively, with substantial enhancement in $D^{*}$ observed at wavelengths near the absorber's bandedge, $\lambda \approx 5 \mu \mathrm{m}$. The TM-polarized response (not shown) is similar to that of the control, with minimal enhancement at features associated with GMR resonances. This is largely a result of the anisotropic absorption coefficient of the T2SL. While we use 1D grating structures here, in order to more clearly identify the optical response associated with the GMR features, future detectors could easily leverage 2D arrays of $\mathrm{GaSb}$ pillars for polarization independent response. 


\section{CONCLUSION}

In conclusion, we have demonstrated enhanced mid-wave infrared $\mathrm{nBn}$ T2SL detectors operating at room temperature (296 K). Leveraging an ultra-thin $\mathrm{nBn}$ detector architecture engineered for MWIR absorption at room temperature, we can substantially reduce detector dark current allowing for high-temperature operation. At the same time, by integrating our detectors into guided-mode resonance structures, we can enhance the response of the detectors to compensate for the reduced absorption coefficient, especially near detector cut-off. We demonstrate TE-polarized peak $\mathrm{EQE} \approx 60 \%$ (46\%) for $\Lambda=1.6 \mu \mathrm{m}(\Lambda=1.8 \mu \mathrm{m})$ grating GMR devices with $>\times 7.5(\times 14)$ enhancement, when compared to the unpatterned control sample, at resonant wavelengths of $\lambda=4.4 \mu \mathrm{m}(\lambda=4.7 \mu \mathrm{m})$ near detector cut-off. We also show estimated peak specific detectivity, $D^{*}=1.2 \times 10^{10}$ and $1 \times 10^{10} \mathrm{~cm} \sqrt{\mathrm{Hz}} / \mathrm{W}$ for the respective grating-patterned detectors, exceeding that of state-of-the-art RT MWIR detectors. The all-epitaxial approach presented here allows us to embed optoelectronic functionality in resonant optical structures, with strongly enhanced performance across the technologically vital MWIR. The ability to efficiently detect, with significant spectral selectivity, the MWIR light with room temperature sensors could have significant implications for gas sensing, thermal imaging, or even free-space communication applications.

\section{ACKNOWLEDGMENT}

This material was based upon work supported by the United States Army under Prime Contract W909MY-20-P-0010. Any opinions, findings, and conclusions or recommendations expressed in this material are those of the author(s) and do not necessarily reflect the views of the U.S. Army. The authors gratefully acknowledge support from the National Science Foundation (ECCS-1926187). Part of the work was done at the University of Texas Microelectronics Research Center (The Texas Nanofabrication Facility), a member of the National Nanotechnology Coordinated Infrastructure (NNCI), supported by the National Science Foundation (No. ECCS2025227).

\section{REFERENCES}

[1] C. H. Grein, M. E. Flatté, J. T. Olesberg, S. A. Anson, L. Zhang, and T. F. Boggess, "Auger recombination in narrow-gap semiconductor superlattices incorporating antimony," J. Appl. Phys., vol. 92, no. 12, pp. 7311-7316, 2002.

[2] H. Mohseni, M. Razeghi, G. J. Brown, and Y. S. Park, "Highperformance InAs/GaSb superlattice photodiodes for the very long wavelength infrared range," Appl. Phys. Lett., vol. 78, no. 15, pp. 21072109, 2001

[3] J. B. Rodriguez, E. Plis, G. Bishop, Y. D. Sharma, H. Kim, L. R. Dawson, and S. Krishna, "nBn structure based on InAs/GaSb type-II strained layer superlattices," Appl. Phys. Lett., vol. 91, no. 4, p. 043514, 2007.

[4] D. Z.-Y. Ting, C. J. Hill, A. Soibel, S. A. Keo, J. M. Mumolo, J. Nguyen, and S. D. Gunapala, "A high-performance long wavelength superlattice complementary barrier infrared detector," Appl. Phys. Lett., vol. 95, no. 2, p. $023508,2009$.

[5] A. Haddadi, S. Adhikary, A. Dehzangi, and M. Razeghi, "Midwavelength infrared heterojunction phototransistors based on type-II InAs/AlSb/GaSb superlattices," Appl. Phys. Lett., vol. 109, no. 2, p. 021107,2016
[6] A. Rogalski, P. Martyniuk, and M. Kopytko, "Type-II superlattice photodetectors versus $\mathrm{HgCdTe}$ photodiodes," Prog. Quantum Electron., vol. 68, p. 100228, 2019.

[7] B. V. Olson, J. K. Kim, E. A. Kadlec, J. F. Klem, S. D. Hawkins, D. Leonhardt, W. T. Coon, T. R. Fortune, M. A. Cavaliere, A. TaukePedretti, and E. A. Shaner, "Minority carrier lifetime and dark current measurements in mid-wavelength infrared $\mathrm{InAs}_{0.91} \mathrm{Sb}_{0.09}$ alloy $\mathrm{nBn}$ photodetectors," Appl. Phys. Lett., vol. 107, no. 18, p. 183504, 2015.

[8] J. A. Nolde, M. Kim, C. S. Kim, E. M. Jackson, C. T. Ellis, J. Abell, O. J. Glembocki, C. L. Canedy, J. G. Tischler, I. Vurgaftman, J. R. Meyer, and E. H. Aifer, "Resonant quantum efficiency enhancement of midwave infrared $\mathrm{nBn}$ photodetectors using one-dimensional plasmonic gratings," Appl. Phys. Lett., vol. 106, no. 26, p. 261109, 2015.

[9] M. D. Goldflam, E. A. Kadlec, B. V. Olson, J. F. Klem, S. D. Hawkins, S. Parameswaran, W. T. Coon, G. A. Keeler, T. R. Fortune, A. TaukePedretti, J. R. Wendt, E. A. Shaner, P. S. Davids, J. K. Kim, and D. W. Peters, "Enhanced infrared detectors using resonant structures combined with thin type-II superlattice absorbers," Appl. Phys. Lett., vol. 109, no. 25, p. 251103, 2016.

[10] V. Letka, A. Bainbridge, A. P. Craig, F. Al-Saymari, and A. R. J. Marshall, "Resonant cavity-enhanced photodetector incorporating a type-ii superlattice to extend mwir sensitivity," Opt. Express, vol. 27, no. 17, pp. 23970-23 980, Aug 2019.

[11] A. Kamboj, L. Nordin, P. Petluru, A. J. Muhowski, D. N. Woolf, and D. Wasserman, "All-epitaxial guided-mode resonance mid-wave infrared detectors," Appl. Phys. Lett., vol. 118, no. 20, p. 201102, 2021.

[12] S. S. Wang and R. Magnusson, "Theory and applications of guidedmode resonance filters," Appl. Opt., vol. 32, no. 14, pp. 2606-2613, May 1993.

[13] A. Szeghalmi, M. Helgert, R. Brunner, F. Heyroth, U. Gösele, and M. Knez, "Tunable Guided-Mode Resonance Grating Filter," Adv. Funct. Mater., vol. 20, no. 13, pp. 2053-2062, 2010.

[14] Y. Zhong, Z. Goldenfeld, K. Li, W. Streyer, L. Yu, L. Nordin, N. Murphy, and D. Wasserman, "Mid-wave infrared narrow bandwidth guided mode resonance notch filter," Opt. Lett., vol. 42, no. 2, pp. 223-226, Jan 2017.

[15] T. Kondo, S. Ura, and R. Magnusson, "Design of guided-mode resonance mirrors for short laser cavities," J. Opt. Soc. Am. A, vol. 32, no. 8, pp. 1454-1458, Aug 2015.

[16] S. Law, D. C. Adams, A. M. Taylor, and D. Wasserman, "Mid-infrared designer metals," Opt. Express, vol. 20, no. 11, pp. 12 155-12 165, May 2012.

[17] E. A. Kadlec, B. V. Olson, M. D. Goldflam, J. K. Kim, J. F. Klem, S. D. Hawkins, W. T. Coon, M. A. Cavaliere, A. Tauke-Pedretti, T. R. Fortune, C. T. Harris, and E. A. Shaner, "Effects of electron doping level on minority carrier lifetimes in n-type mid-wave infrared InAs/InAs1xSbx type-II superlattices," Appl. Phys. Lett., vol. 109, no. 26, p. 261105 2016.

[18] "From V.A. Podolskiy's research group, see ." [Online]. Available: http://faculty.uml.edu/vpodolskiy/codes/index.html for an implementation of Rigorous Coupled Wave Analysis

[19] T. Manyk, K. Michalczewski, K. Murawski, P. Martyniuk, and J. Rutkowski, "InAs/InAsSb Strain-Balanced Superlattices for Longwave Infrared Detectors," Sensors, vol. 19, no. 8, 2019.

[20] D. Zuo, R. Liu, D. Wasserman, J. Mabon, Z.-Y. He, S. Liu, Y.H. Zhang, E. A. Kadlec, B. V. Olson, and E. A. Shaner, "Direct minority carrier transport characterization of InAs/InAsSb superlattice nBn photodetectors," Appl. Phys. Lett., vol. 106, no. 7, p. 071107, 2015.

[21] A. Chandola, R. Pino, and P. S. Dutta, "Below bandgap optical absorption in tellurium-doped GaSb," Semiconductor Science and Technology, vol. 20, no. 8, pp. 886-893, jul 2005.

[22] W. E. Tennant, D. Lee, M. Zandian, E. Piquette, and M. Carmody, "MBE HgCdTe Technology: A Very General Solution to IR Detection, Described by "Rule 07", a Very Convenient Heuristic," J. Electron. Mater., vol. 37, no. 9, pp. 1406-1410, Sep. 2008.

[23] D. Lee, P. Dreiske, J. Ellsworth, R. Cottier, A. Chen, S. Tallaricao, A. Yulius, M. Carmody, E. Piquette, M. Zandian, and S. Douglas, "Law 19: The ultimate photodiode performance metric," SPIE, vol. 11407, pp. $93-105,2020$.

[24] A. P. Craig, V. Letka, M. Carmichael, T. Golding, and A. R. Marshall, "Inassb-based detectors on gasb for near-room-temperature operation in the mid-wave infrared," Applied Physics Letters, vol. 118, no. 25, p. 251103, 2021.

[25] J. Kim, H. Yuan, J. Kimchi, J. Lei, E. Rangel, P. Dreiske, and A. Ikhlassi, "HOT MWIR InAs/InAsSb T2SL discrete photodetector development," in Proc. SPIE, vol. 10624, 2018, pp. $108-115$. 